\title{
Multiplexing FLIM for High Content Screening Applications
}

\author{
Qiyin Fang \\ Canada Research Chair in Biophotonics \\ McMaster University \\ Canada
}

In high content screening (HCS) of drug leads, throughput is a critical requirement. We developed a FLIM technique, which utilizes lenslet arrays and a multiplexing confocal scanning process. This technique achieves high throughput without sacrificing resolution, ideal for HCS. The performance of the system in terms of cross-talk, lifetime estimation, and resolution is characterized through live cell imaging experiments. 